\title{
A novel effective method for the assessment of microvascular function in male patients with coronary artery disease: a pilot study using laser speckle contrast imaging
}

\author{
J.P. Borges ${ }^{1}$, G.O. Lopes ${ }^{1,2}$, V. Verri², M.P. Coelho ${ }^{2}$, P.M.C. Nascimento ${ }^{2}$, D.A. Kopiler ${ }^{2}$ \\ and E. Tibirica ${ }^{2,3}$ \\ ${ }^{1}$ Laboratório de Atividade Física e Promoção à Saúde, Departamento de Desporto Coletivo, \\ Instituto de Educação Física e Desportos, Universidade do Estado do Rio de Janeiro, Rio de Janeiro, RJ, Brasil \\ ${ }^{2}$ Instituto Nacional de Cardiologia, Rio de Janeiro, RJ, Brasil \\ ${ }^{3}$ Laboratório de Investigação Cardiovascular, Departamento Osório de Almeida, Instituto Oswaldo Cruz, FIOCRUZ, \\ Rio de Janeiro, RJ, Brasi
}

\begin{abstract}
Evaluation of microvascular endothelial function is essential for investigating the pathophysiology and treatment of cardiovascular and metabolic diseases. Although laser speckle contrast imaging technology is well accepted as a noninvasive methodology for assessing microvascular endothelial function, it has never been used to compare male patients with coronary artery disease with male age-matched healthy controls. Thus, the aim of this study was to determine whether laser speckle contrast imaging could be used to detect differences in the systemic microvascular functions of patients with established cardiovascular disease $(n=61)$ and healthy age-matched subjects $(n=24)$. Cutaneous blood flow was assessed in the skin of the forearm using laser speckle contrast imaging coupled with the transdermal iontophoretic delivery of acetylcholine and postocclusive reactive hyperemia. The maximum increase in skin blood flow induced by acetylcholine was significantly reduced in the cardiovascular disease patients compared with the control subjects $(74$ vs $116 \% ; \mathrm{P}<0.01)$. With regard to post-occlusive reactive hyperemia-induced vasodilation, the patients also presented reduced responses compared to the controls $(0.42 \pm 0.15$ vs $0.50 \pm 0.13 \mathrm{APU} / \mathrm{mmHg} ; \mathrm{P}=0.04)$. In conclusion, laser speckle contrast imaging can identify endothelial and microvascular dysfunctions in male individuals with cardiovascular disease. Thus, this technology appears to be an efficient non-invasive technique for evaluating systemic microvascular and endothelial functions, which could be valuable as a peripheral marker of atherothrombotic diseases in men.
\end{abstract}

Key words: Ischemic heart disease; Cutaneous vascular conductance; Microvascular flowmetry; Endothelial function; Postocclusive reactive hyperemia

\section{Introduction}

Cardiovascular diseases are among the leading causes of morbidity and mortality worldwide (1). Atherosclerosis is a major disease that can lead to ischemia of the heart, brain or extremities, resulting in organ damage or infarction. The pathophysiology of atherosclerosis comprises a series of highly specific cellular and molecular responses that can be defined as an inflammatory disease and may be present throughout a person's lifetime (2). Endothelial dysfunction precedes clinically detectable atherosclerosis and can also contribute to arterial lesion development and later clinical complications (3). Therefore, the evaluation of microvascular endothelial function is essential for investigating the pathophysiology of cardiometabolic diseases, including arterial hypertension, diabetes, dyslipidemia and obesity (4).

Nonetheless, from a clinical perspective, the development of an easy-to-perform and noninvasive test for routinely assessing microvascular endothelial function is still required. An optimal tool for routine use should, among other factors, be non-invasive, specific, and able to detect diseased patients (3). In this sense, the more common non-invasive methods that have been developed in clinical microvascular laboratories are based on laser technology, such as laser Doppler imaging and

Correspondence: E. Tibirica: <etibi@ioc.fiocruz.br>

Received June 3, 2016| Accepted July 7, 2016 
laser Doppler flowmetry (4-6). However, measuring microvasculature function presents a particular challenge because the vessel structure is spatially inhomogeneous, and perfusion can be highly variable over time (6). Previous studies have suggested that these features are related to the high variability of measurements based on single-point methods, including laser Doppler technology measurements $(5,7,8)$.

Recently, another non-invasive method for measuring microvascular blood flow termed laser speckle contrast imaging (LSCl) has gained increasing attention. This methodology has previously been used to evaluate blood flow in experimental settings (9-11) and in the human brain $(12,13)$. The $\mathrm{LSCl}$ method was first introduced in the 1980s and is a powerful tool for the full-field imaging of blood flow (14). In contrast to laser Doppler technologies, this method consists of assessing the blood flow response over a broad area of analysis rather than at a single point (5). This broad area of analysis is particularly important because it reduces the variability of the measurements due to the spatial heterogeneity of the skin microvasculature, especially in response to drug delivery (5). Indeed, previous studies have shown that the reproducibility of $\mathrm{LSCl}$ coupled with post-occlusive reactive hyperemia $(\mathrm{PORH})(5)$ and the iontophoresis of acetylcholine are excellent (3).

Many studies have examined the methodological issues of LSCI (15-17) and compared different methods in terms of their abilities to assess blood flow in healthy individuals $(3,18,19)$. Nonetheless, previous data regarding the ability of $\mathrm{LSCl}$ to detect diseased patients are limited $(4,20)$. Thus, our aim was to determine whether $\mathrm{LSCl}$ is an efficient method for identifying impairments in skin microvascular function in male patients with coronary artery disease (CAD) compared to age-matched healthy individuals.

\section{Material and Methods}

\section{Ethics}

All procedures described in the present study were conducted in accordance with the Declaration of Helsinki of 1975 as revised in 2000 and were approved by the Institutional Review Board (IRB) of the Instituto Nacional de Cardiologia do Rio de Janeiro, Brazil (protocol \#894.911). Once considered eligible, all subjects signed an informed consent form that was approved by the IRB.

\section{Subjects}

A sample of 61 consecutive male patients with CAD $(58.9 \pm 12.8$ years old) from the Cardiac Rehabilitation Program of the Instituto Nacional de Cardiologia, Rio de Janeiro, Brazil and 24 healthy male individuals $(56.1 \pm 4.9$ years old), randomly recruited from the staff of the same institution, were enrolled in this cross-sectional study.
In the CAD patients, $67 \%$ had previous acute myocardial infarction and referred diagnoses of diabetes (44\%), arterial hypertension (80\%), and dyslipidemia (65\%) according to their medical records. In the control subjects, all of the abovementioned pathologies were absent. CAD was defined by the occurrence of any acute coronary syndrome, including ST and non-ST elevation myocardial infarction, and unstable angina (all defined by characteristic histories and electrocardiographic and cardiac enzyme abnormalities) or by the diagnosis of obstructive CAD based on coronary angiography (defined as $\geqslant 50 \%$ stenosis of any epicardial coronary artery) in patients with stable angina.

According to the study protocol, the patients and controls underwent cutaneous microvascular reactivity testing. On the morning scheduled for the test, the patients presented in a 12-h fasted condition for blood collection. The patients must not have smoked or ingested caffeine from the night before until the completion of the tests. The patients took their usual medications on the morning of the tests, except direct vasodilators, which were administered immediately after the microcirculatory tests.

\section{Evaluation of skin microvascular reactivity using LSCI}

Microcirculatory tests were performed after a 20-min rest with the patients in the supine position in a temperature-controlled room $\left(23 \pm 1^{\circ} \mathrm{C}\right)$ approximately $1 \mathrm{~h}$ after a light breakfast. Microvascular reactivity was evaluated using a LSCI system with a laser wavelength of $785 \mathrm{~nm}$ (PeriCam PSI system, Perimed, Sweden) in combination with the iontophoresis of acetylcholine (ACh) for noninvasive and continuous measurements of cutaneous microvascular perfusion changes (in arbitrary perfusion units, APU; Figure 1) $(4,20)$. The image acquisition rate was 8 images/s, and the distance between the laser head and the skin surface was fixed at $20 \mathrm{~cm}$ as recommended in the manufacturer's manual. Images were analyzed using the manufacturer's software (PIMSoft, Perimed).

The skin sites used for the microvascular flow recordings were randomly chosen on the ventral surface of the forearm, and hair, broken skin, areas of skin pigmentation and visible veins were avoided. The drugdelivery electrode was secured using an adhesive disc (LI 611, Perimed). Two measurement areas (circular regions of interest) of approximately $80 \mathrm{~mm}^{2}$ were examined. One of the measurement areas was within the electrode area (for the ACh-related measurements), and the other was adjacent to the electrode (for the PORH measurements). A vacuum cushion (a specially constructed pillow filled with polyurethane foam that can be molded to any desired shape by creating a vacuum; from AB Germa, Sweden) was used to reduce recording artifacts generated by arm movements. ACh ( $2 \% \mathrm{w} / \mathrm{v}$; Sigma Chemical Co., USA) iontophoresis was performed using a micropharmacology system (PF 751 Perilont USB Power Supply, Perimed) 

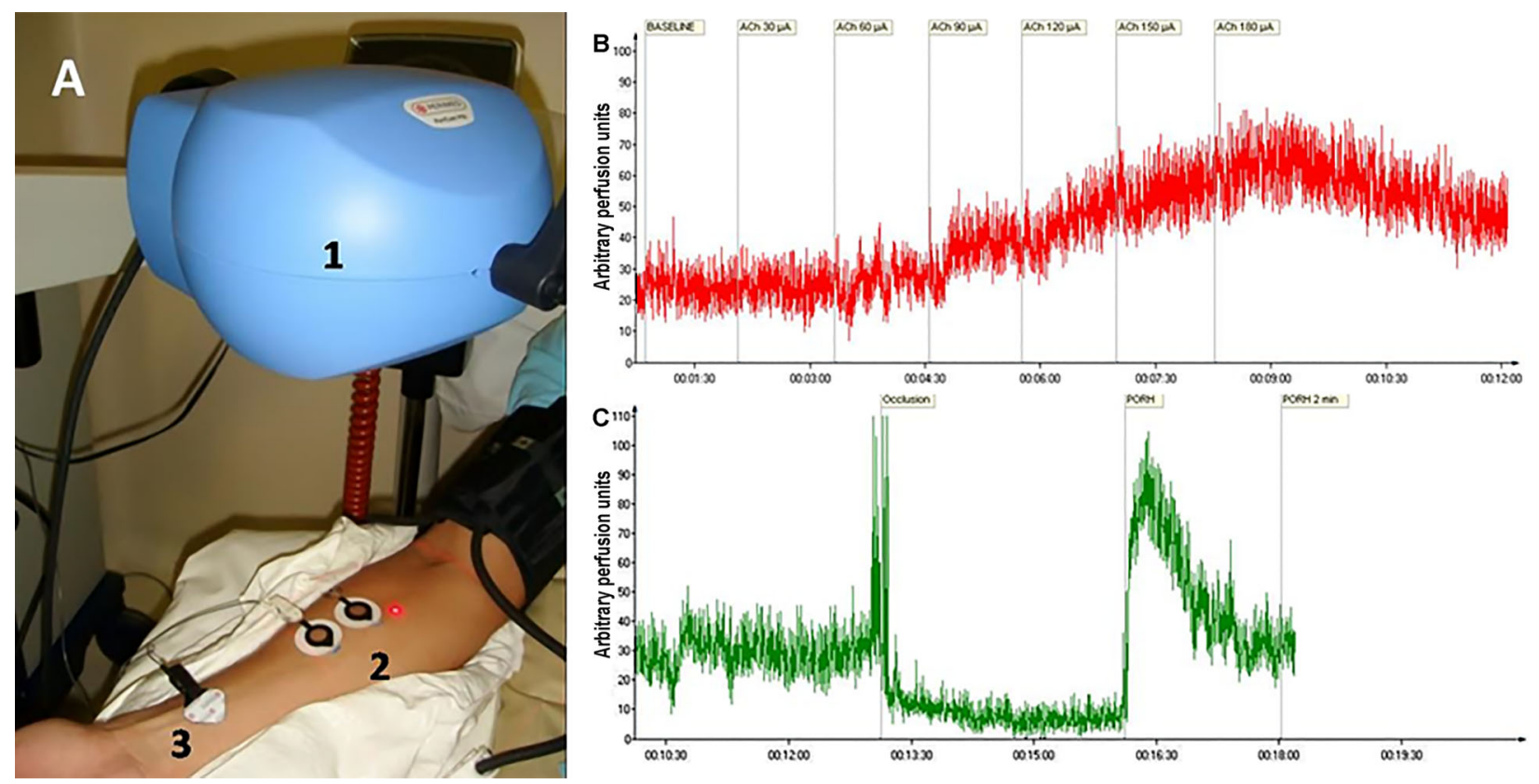

Figure 1. $A$, Photograph of the experimental set up used in the assessment of skin microvascular perfusion using laser speckle contrast imaging coupled with iontophoresis of vasodilator drugs. 1) Imager head, 2) drug-delivery iontophoresis electrodes, 3) dispersive electrode (see Material and Methods). $B$, Representative examples of the effects of the transdermal iontophoretic delivery of cumulative doses of acetylcholine $(\mathrm{ACh})$ on skin blood flow during the iontophoresis of $\mathrm{ACh}(2 \% \mathrm{w} / \mathrm{v})$ using increasing anodal currents of $30,60,90$, 120,150 , and $180 \mu \mathrm{A}$ applied in 10-s intervals spaced $1 \mathrm{~min}$ apart. $C$, skin microvascular blood flow during post-occlusive reactive hyperemia $(\mathrm{PORH})$.

with increasing anodal currents of $30,60,90,120,150$, and $180 \mu \mathrm{A}$ applied in 10-s intervals spaced 1 min apart (the total charges were $0.3,0.6,0.9,1.2,1.5$, and $1.8 \mathrm{mC}$, respectively). The dispersive electrode was attached approximately $15 \mathrm{~cm}$ away from the electrophoresis chamber. Of note, the drug was not injected but rather was placed in contact with the skin surface.

During the PORH test, arterial occlusion was performed with suprasystolic pressure $(50 \mathrm{mmHg}$ above the systolic arterial pressure) using a sphygmomanometer over $3 \mathrm{~min}$. Following the release of the pressure, the maximum flux was measured. The measurements of skin blood flow were divided by the mean arterial pressure to yield the cutaneous vascular conductance (CVC) in $\mathrm{APU} / \mathrm{mmHg}$. The amplitudes of the PORH responses were expressed as the peak CVC minus the baseline CVC.

\section{Statistical analysis}

The results are reported as means $\pm S D$. Normal sample distributions were confirmed with the Shapiro-Wilk test. Comparisons between groups were performed using the two-tailed unpaired Student's t-test. The null hypothesis was rejected at $P<0.05$. The Prism version 5.0 statistical package (GraphPad Software Inc., USA) was employed.

\section{Results}

\section{Subject characteristics}

Baseline clinical characteristics are presented in Table 1. The healthy volunteers exhibited higher values of the total, LDL and HDL cholesterol and lower glucose values compared with the CAD patients. Concerning the use of cardiovascular drugs, $28 \%$ of the CAD patients used angiotensin-converting enzyme inhibitors, $90 \%$ used $\beta$-blockers, 92\% used lipid-lowering drugs, 36\% used nitrates, $52 \%$ used angiotensin II receptor blockers, and $57 \%$ used diuretics.

\section{Skin microvascular reactivity}

The mean resting CVC did not differ between the groups (healthy subjects: $0.27 \pm 0.06$ vs CAD patients: $0.29 \pm 0.08 \mathrm{APU} / \mathrm{mmHg} ; P=0.21$ ). The patients' microvascular responses to $\mathrm{ACh}$ were significantly reduced compared with those of the healthy subjects; the maximum increase in CVC induced by ACh in the patients was $74 \%$ compared with $116 \%$ in the controls $(P=0.002)$. The increases in CVC relative to baseline during the iontophoresis of $\mathrm{ACh}$ were $0.31 \pm 0.14$ and $0.19 \pm 0.14$ APU/mmHg in the control and CAD participants, respectively $(P=0.001$; Figure $2 A)$. The areas under the curves for the ACh-induced vasodilation were $7005 \pm 4493$ and 
Table 1. Baseline clinical characteristics of the male coronary artery disease (CAD) patients and healthy controls.

\begin{tabular}{lccr}
\hline & Healthy controls $(\mathrm{n}=24)$ & CAD patients $(\mathrm{n}=61)$ & $\mathrm{P}$ \\
\hline Age (years) & $56.2 \pm 4.9$ & $58.9 \pm 12.8$ & 0.31 \\
Body weight $(\mathrm{kg})$ & $77.7 \pm 11.9$ & $78.8 \pm 13.2$ & 0.90 \\
Body mass index $\left(\mathrm{kg} / \mathrm{m}^{2}\right)$ & $25.9 \pm 3.3$ & $27.4 \pm 3.7$ & 0.11 \\
Smokers, $\mathrm{n}(\%)$ & $3(12.5)$ & $6(9.8)$ & 0.70 \\
Systolic blood pressure $(\mathrm{mmHg})$ & $133.1 \pm 12.1$ & $134.8 \pm 19.4$ & 0.70 \\
Diastolic blood pressure $(\mathrm{mmHg})$ & $79.3 \pm 7.4$ & $76.2 \pm 10.3$ & 0.18 \\
Mean blood pressure $(\mathrm{mmHg})$ & $97.3 \pm 8.4$ & $95.7 \pm 12.2$ & 0.57 \\
Fasting plasma glucose $(\mathrm{mg} / \mathrm{dL})$ & $99.48 \pm 7.39$ & $116.1 \pm 21.7$ & $<0.01$ \\
GTO (Ul/L) & $24.27 \pm 6.09$ & $22.4 \pm 7.5$ & 0.48 \\
GPT (Ul/L) & $29.67 \pm 12.66$ & $25.2 \pm 9.5$ & 0.18 \\
Total cholesterol $(\mathrm{mg} / \mathrm{dL})$ & $217.36 \pm 26.44$ & $162.8 \pm 48.6$ & $<0.01$ \\
LDL- cholesterol $(\mathrm{mg} / \mathrm{dL})$ & $142.91 \pm 25.84$ & $93.5 \pm 37.2$ & $<0.01$ \\
HDL-cholesterol $(\mathrm{mg} / \mathrm{dL})$ & $44.91 \pm 8.16$ & $38.2 \pm 10.6$ & $<0.01$ \\
Triglycerides $(\mathrm{mg} / \mathrm{dL})$ & $147.64 \pm 65.81$ & $144.3 \pm 85.8$ & 0.45 \\
\hline
\end{tabular}

Results are reported as means \pm SD. GTO: glutamic oxaloacetic transaminase; GPT: glutamic pyruvic transaminase. $\mathrm{P}$ values were estimated using two-tailed unpaired $t$-tests or Fisher's exact test, where appropriate.
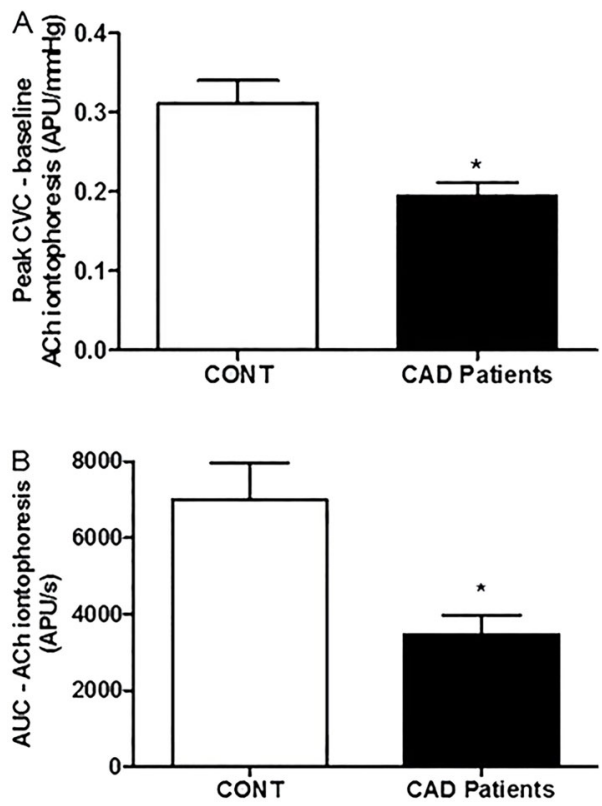

Figure 2. Effects of the skin iontophoresis of acetylcholine (ACh) on cutaneous microvascular conductance [CVC, expressed in arbitrary perfusion units (APU) divided by mean arterial pressure in $\mathrm{mmHg}$ in healthy volunteers (CONT) and male patients with coronary artery disease (CAD). A, peak CVC minus baseline CVC resulting from the iontophoresis of $A C h$. $B$, area under the curve (AUC) for the iontophoresis of ACh on the skin. Data are reported as means $\pm \mathrm{SE}$. ${ }^{*} \mathrm{P}<0.05$, compared to control (two-tailed unpaired Student's $t$-test).

$3469 \pm 3075 \mathrm{APU} / \mathrm{s}$ in the control and CAD participants, respectively $(P=0.001$; Figure $2 B)$.

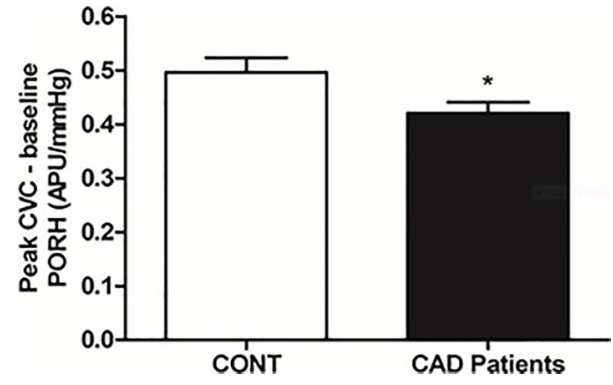

Figure 3. Effects of post-occlusive reactive hyperemia $(\mathrm{PORH})$ on skin cutaneous microvascular conductance [CVC, expressed in arbitrary perfusion units (APU) divided by mean arterial pressure in $\mathrm{mmHg}$ in healthy volunteers (CONT) and male patients with coronary artery disease (CAD). ${ }^{*} P<0.05$, compared to control (two-tailed unpaired Student's $t$-test).

Concerning the microvascular reactivity to $\mathrm{PORH}$, the CAD patients also presented lower responses than the healthy participants $(0.42 \pm 0.15$ and $0.50 \pm 0.13 \mathrm{APU} /$ $\mathrm{mmHg}$, respectively; $\mathrm{P}=0.04$; Figure 3 ).

\section{Discussion}

The main finding of this study is that $\mathrm{LSCl}$ is capable of identifying the reduced endothelium-dependent skin microvascular vasodilator responses in patients with CAD compared with healthy subjects.

Our results are relevant to medical research because the evaluation of microvascular endothelial function is essential for investigating the pathophysiology of cardiometabolic diseases, including arterial hypertension and diabetes (4). In this context, LSCI is a recently developed 
technique that is based on laser speckle contrast analysis (LASCA) and provides an index of microvascular blood flow (7). LASCA is based on the principle that when an object is illuminated by a coherent light, such as laser, the light will be scattered by a collection of randomly distributed particles to produce a characteristic random interference pattern known as a speckle pattern that consists of light and dark areas (21). If the illuminated object is static, the speckle pattern is stationary. When there is object movement, such as red blood cells in a tissue, the speckle pattern will change overtime. The level of blurring will differ according to the degree of movement; greater movement elicits greater blurring of the speckle pattern. The level of blurring is quantified by the speckle contrast. By acquiring an image of the speckle pattern and quantifying the blurring of the speckles via measurements of the spatial intensity variations in the speckle pattern, spatial maps of the relative blood flow can be obtained $(21,22)$.

Because of its high level of reproducibility and advantages of good spatial and temporal resolutions, LSCI plays a key role in the analysis of microvascular function, especially when coupled with $\mathrm{PORH}$ and non-invasive transdermal drug delivery (iontophoresis) $(6,7,23)$. It is also noteworthy that the cutaneous microcirculation is now considered to be an accessible and representative vascular bed for assessments of systemic microcirculatory reactivity $(8,24,25)$. Moreover, alterations of microvascular function in the skin have previously been demonstrated to correlate with increased CAD risk (26).

Many investigations have focused on verifying the day-to-day repeatability and inter-subject reproducibility of $\mathrm{LSCl}$ and comparing different methodologies in terms of the interpretations of the experimental results $(3,5,11$, $18,19,27)$. Nevertheless, only two studies have compared the systemic microvascular endothelial function of patients with that of healthy individuals using $\operatorname{LSCl}(4,20)$. The study by Cordovil et al. (4) was the first to validate this methodology for the evaluation of cardiovascular and metabolic diseases. These authors first suggested that $\mathrm{LSCl}$ could be a useful noninvasive technique for evaluating systemic microvascular endothelial function. These authors demonstrated that patients with arterial hypertension and severe dyslipidemia exhibit a reduction of $47 \%$ in the maximum increase in systemic microvascular blood flow induced by ACh compared with controls (4). In the present study, this response in CAD patients was reduced by $36 \%$ compared with the control subjects. The difference in the results between studies is probably attributable to the fact that the previous study did not

\section{References}

1. Go AS, Mozaffarian D, Roger VL, Benjamin EJ, Berry JD, Blaha MJ, et al. Heart disease and stroke statistics - 2014 update: compare patients with an age-matched control group. In addition to cardiovascular diseases, age differences between groups undoubtedly contributed to the observed endothelial dysfunction. Indeed, aging is well known to be associated with a progressive deterioration in endothelial function (28). Thus, a major strength of the current study compared with previous research is that LSCl was used for the first time to compare male patients with established CAD and healthy age-matched subjects. Although LSCI technology had been previously validated (4), we demonstrated that $\mathrm{LSCl}$ is still an efficient tool for the detection of endothelial dysfunction even when patients with compromised microvascular endothelial function are compared with healthy counterparts of the same gender and age.

A previous study from our group compared the skin microvascular functions in individuals with early-onset CAD and healthy individuals using $\mathrm{LSCl}$ (20). Our results agree with the results of Souza et al. (20) and demonstrate that the endothelium-dependent skin microvascular vasodilator responses induced by both $\mathrm{ACh}$ and $\mathrm{PORH}$ are significantly reduced in patients with premature CAD compared with agematched healthy individuals. These latter results suggest that $\mathrm{LSCl}$ could become a useful non-invasive technology for the development of early markers of microvascular endothelial dysfunction in cardiovascular disease.

The absence of women in our sample is the major limitation of the present study. Cutaneous blood flow may vary according to the hormonal oscillations of the menstrual cycle and therefore interfere with $\mathrm{LSCl}$ data. This is important because the present results should be interpreted with caution when evaluating women, especially those who did not achieve menopause age.

In conclusion, our data indicated that $\mathrm{LSCl}$ was capable of identifying endothelial microvascular dysfunctions in male individuals who present cardiovascular diseases. Thus, LSCI appears to be an efficient noninvasive technique for evaluating systemic microvascular and endothelial functions, which could be valuable as a peripheral marker of atherothrombotic diseases in men. Further research investigating its use in women with cardiovascular diseases is certainly warranted.

\section{Acknowledgments}

The authors would like to thank Marcio Marinho Gonzalez for his excellent technical assistance. This work was partially supported by grants from CNPq (National Council of Scientific and Technological Research) and FAPERJ (Research Support Foundation of the State of Rio de Janeiro).

a report from the American Heart Association. Circulation 2014; 129: e28-e292, doi: 10.1161/01.cir.0000441139.02102.80. 
2. Ross R. Atherosclerosis - an inflammatory disease. N Engl J Med 1999; 340: 115-126, doi: 10.1056/NEJM1999011434 00207.

3. Puissant $C$, Abraham $P$, Durand $S$, Humeau-Heurtier A, Faure $S$, Leftheriotis $G$, et al. Reproducibility of non-invasive assessment of skin endothelial function using laser Doppler flowmetry and laser speckle contrast imaging. PLOS One 2013; 8: e61320, doi: 10.1371/journal.pone.0061320.

4. Cordovil I, Huguenin G, Rosa G, Bello A, Kohler O, de Moraes $\mathrm{R}$, et al. Evaluation of systemic microvascular endothelial function using laser speckle contrast imaging. Microvasc Res 2012; 83: 376-379, doi: 10.1016/j.mvr.2012.01.004.

5. Roustit M, Millet C, Blaise S, Dufournet B, Cracowski JL. Excellent reproducibility of laser speckle contrast imaging to assess skin microvascular reactivity. Microvasc Res 2010; 80: 505-511, doi: 10.1016/j.mvr.2010.05.012.

6. Allen J, Howell K. Microvascular imaging: techniques and opportunities for clinical physiological measurements. Physiol Meas 2014; 35: R91-R141, doi: 10.1088/0967-3334/35/7/R91.

7. Roustit M, Cracowski JL. Non-invasive assessment of skin microvascular function in humans: an insight into methods. Microcirculation 2012; 19: 47-64, doi: 10.1111/j.1549-8719. 2011.00129.x.

8. Roustit M, Cracowski JL. Assessment of endothelial and neurovascular function in human skin microcirculation. Trends Pharmacol Sci 2013; 34: 373-384, doi: 10.1016/ j.tips.2013.05.007.

9. Takeshima Y, Miyake H, Nakagawa I, Motoyama Y, Park YS, Nakase $\mathrm{H}$. Visualization of regional cerebral blood flow dynamics during cortical venous occlusion using laser speckle contrast imaging in a rat model. J Stroke Cerebrovasc Dis 2015; 24: 2200-2206, doi: 10.1016/j.jstrokecerebrovasdis. 2015.04.048.

10. Winship IR. Laser speckle contrast imaging to measure changes in cerebral blood flow. Methods Mol Biol 2014; 1135: 223-235, doi: 10.1007/978-1-4939-0320-7.

11. Dufour S, Atchia Y, Gad R, Ringuette D, Sigal I, Levi O. Evaluation of laser speckle contrast imaging as an intrinsic method to monitor blood brain barrier integrity. Biomed Opt Express 2013; 4: 1856-1875, doi: 10.1364/BOE.4.001856.

12. Parthasarathy $A B$, Weber EL, Richards LM, Fox DJ, Dunn AK. Laser speckle contrast imaging of cerebral blood flow in humans during neurosurgery: a pilot clinical study. J Biomed Opt 2010; 15: 066030, doi: 10.1117/1.3526368.

13. Richards LM, Towle EL, Fox DJ Jr, Dunn AK. Intraoperative laser speckle contrast imaging with retrospective motion correction for quantitative assessment of cerebral blood flow. Neurophotonics 2014; 1: 015006, doi: 10.1117/1.NPh. 1.1.015006.

14. Boas DA, Dunn AK. Laser speckle contrast imaging in biomedical optics. J Biomed Opt 2010; 15: 011109, doi: 10. 1117/1.3285504.

15. Richards LM, Kazmi SM, Davis JL, Olin KE, Dunn AK. Low-cost laser speckle contrast imaging of blood flow using a webcam. Biomed Opt Express 2013; 4: 2269-2283, doi: 10.1364/BOE.4.002269.

16. Zakharov P, Volker AC, Wyss MT, Haiss F, Calcinaghi N, Zunzunegui $\mathrm{C}$, et al. Dynamic laser speckle imaging of cerebral blood flow. Opt Express 2009; 17: 13904-13917, doi: 10.1364/OE.17.013904.

17. Ponticorvo A, Dunn AK. How to build a Laser Speckle Contrast Imaging (LSCl) system to monitor blood flow. $J$ Vis Exp 2010, doi: 10.3791/2004.

18. Humeau-Heurtier A, Abraham P, Mahe G. Analysis of laser speckle contrast images variability using a novel empirical mode decomposition: comparison of results with laser Doppler flowmetry signals variability. IEEE Trans Med Imaging 2015; 34 : 618-627, doi: 10.1109/TMl.2014.2364079.

19. Humeau-Heurtier A, Abraham $P$, Mahe G. Linguistic analysis of laser speckle contrast images recorded at rest and during biological zero: comparison with laser Doppler flowmetry data. IEEE Trans Med Imaging 2013; 32: 2311-2321, doi: 10.1109/TMI.2013.2281620.

20. Souza EG, De Lorenzo A, Huguenin G, Oliveira GM, Tibirica E. Impairment of systemic microvascular endothelial and smooth muscle function in individuals with early-onset coronary artery disease: studies with laser speckle contrast imaging. Coron Artery Dis 2014; 25: 23-28, doi: 10.1097/ MCA.0000000000000055.

21. Briers JD, Webster S. Laser speckle contrast analysis (LASCA): a nonscanning, full-field technique for monitoring capillary blood flow. J Biomed Opt 1996; 1: 174-179, doi: 10.1117/12.231359.

22. Dunn AK, Bolay $H$, Moskowitz MA, Boas DA. Dynamic imaging of cerebral blood flow using laser speckle. J Cereb Blood Flow Metab 2001; 21: 195-201, doi: 10.1097/ 00004647-200103000-00002.

23. Humeau-Heurtier A, Guerreschi E, Abraham P, Mahe G. Relevance of laser Doppler and laser speckle techniques for assessing vascular function: state of the art and future trends. IEEE Trans Biomed Eng 2013; 60: 659-666, doi: 10.1109/TBME.2013.2243449.

24. Holowatz LA, Thompson-Torgerson CS, Kenney WL. The human cutaneous circulation as a model of generalized microvascular function. J Appl Physiol 2008; 105: 370-372, doi: 10.1152/japplphysiol.00858.2007.

25. Rizzoni D, Aalkjaer C, De Ciuceis C, Porteri E, Rossini C, Rosei CA, et al. How to assess microvascular structure in humans. High Blood Press Cardiovasc Prev 2011; 18 : 169-177, doi: 10.2165/11593640-000000000-00000.

26. IJzerman RG, de Jongh RT, Beijk MA, van Weissenbruch MM, Delemarre-van de Waal HA, Serne EH, et al. Individuals at increased coronary heart disease risk are characterized by an impaired microvascular function in skin. Eur J Clin Invest 2003; 33: 536-542, doi: 10.1046/j.1365-2362.2003.01179.x.

27. Huguenin GV, Moreira AS, Siant'Pierre TD, Goncalves RA, Rosa G, Oliveira GM, et al. Effects of dietary supplementation with Brazil nuts on microvascular endothelial function in hypertensive and dyslipidemic patients: A randomized crossover placebo-controlled trial. Microcirculation 2015; 22: 687-699, doi: 10.1111/micc.12225.

28. Khalil A, Humeau-Heurtier A, Mahe G, Abraham P. Laser speckle contrast imaging: age-related changes in microvascular blood flow and correlation with pulse-wave velocity in healthy subjects. J Biomed Opt 2015; 20: 051010, doi: 10.1117/1.JBO.20.5.051010. 